\title{
Unique metastases of ALK mutated lung cancer activated to the adnexa of the uterus
}

\author{
Allison H. West ${ }^{1}$, S. Diane Yamada ${ }^{2}$, Heber MacMahon ${ }^{3}$, Sheetal S. Acharya ${ }^{4}$, Siraj M. Ali ${ }^{5}$, J ie $\mathrm{He}^{5}$, \\ Rimas V. Lukas ${ }^{6}$, Vincent A. Miller ${ }^{5}$, Ravi Salgia ${ }^{1}$ \\ 1. Department of Medicine, The University of Chicago, Chicago, IL, United States. 2. Department of Obstetrics/ \\ Gynecology, The University of Chicago, Chicago, IL, United States. 3. Department of Radiology, The University of Chicago, \\ Chicago, IL, United States. 4. Marquette General Hematology Oncology, Marquette, MI, United States. 5. Foundation \\ Medicine, Cambridge, MA, United States. 6. Department of Neurology, University of Chicago, Chicago, IL, United States.
}

Correspondence: Ravi Salgia, M.D., Ph.D. Address: 5841 S. Maryland Avenue, Chicago, IL 60637, United States. E-mail: rsalgia@medicine.bsd.uchicago.edu

Received: May 12, 2014

DOI : $10.5430 /$ crcp.v1n2p151
Accepted: June 27, 2014

Online Published: July 8, 2014

URL: http://dx.doi.org/10.5430/crcp.v1n2p151

\begin{abstract}
Anaplastic lymphoma kinase (ALK) fusion positive non-small cell lung cancer (NSCLC) is a relatively novel molecular subtype in lung cancer. ALK targeted therapies such as crizotinib increase survival benefit in this patient population. However, further understanding of the disease subtype including patterns of metastasis and pathways of treatment resistance are needed. We describe a 50 year-old-woman diagnosed with stage IV NSCLC, T2N3M1b, ALK fusion positive disease. Although her initial sites of metastasis included the liver and bone, her story is notable for crizotinib resistant growth of a metastatic lesion to her adnexa. This report brings to attention unique mechanisms of metastasis in ALK positive patients.
\end{abstract}

\section{Key words}

Non-small cell lung cancer, Adnexa, Metastasis, Anaplastic lymphoma kinase

\section{Case presentation}

A 50-year-old woman was referred to our Thoracic Oncology Group for metastatic Anaplastic lymphoma kinase (ALK) fusion positive adenocarcinoma of the lung. She initially presented to her primary care physician in September 2009 with a known history of asthma and persistent wheezing. CT imaging demonstrated a left lung mass, diagnosed as grade 3 adenocarcinoma of the lung, EGFR non-mutated on biopsy. Liver biopsy confirmed metastatic disease and PET also noted metastasis to the bone. She was initially treated with pemetrexed, carboplatin, and bevacizumab with partial response through February 2010 followed by maintenance. In June 2011, PET showed recurrence of bone disease and the decision was made to pursue novel therapeutic options. The patient underwent ALK fusion testing, which was positive by fluorescence in-situ hybridization (FISH) analysis using Vysis ALK break-apart FISH probe kit. In August 2011, she was referred to our Thoracic Oncology Clinic for crizotinib therapy. Treatment was initially delayed after screening brain MRI demonstrated metastases; she completed whole brain radiation therapy and started off protocol crizotinib with brief interruption for stereotactic radiosurgery to recurrent brain metastasis. In November 2012, PET showed an enlarging ovary and progressive brain disease for which she again underwent stereotactic radiosurgery. She was continued on systemic 
therapy until April 2013 when restaging identified decreased tumor burden with the exception of the previously noted adnexal mass, now concerning for metastatic disease. The lesion was described as a heterogeneous enhancing mixed cystic and solid adnexal mass measuring $12.8 \mathrm{~cm} \times 11.7 \mathrm{~cm}$ on axial view and $12.2 \mathrm{~cm}$ on cranial caudal view (see Figure 1). In May 2013, she was referred to gynecological oncology for abdominal hysterectomy with bilateral salpingo-oophorectomy. Pathology revealed left and right ovaries with metastatic adenocarcinoma ALK-fusion positive, left and right fallopian tubes without diagnostic abnormality, 0/13 pelvic nodes positive for tumor, uterus and cervix without diagnostic abnormality, and 2/11 peri-aortic lymph nodes with metastatic adenocarcinoma (see Figure 2, 3). Following surgery, the decision was made to restart ALK therapy after concluding that the ovarian metastases were a potential sanctuary site. At this time, a tissue sample was also sent to Foundation One for further molecular analysis and to identify treatment options, however no additional treatment specific genomic alterations were noted (see Figure 4).
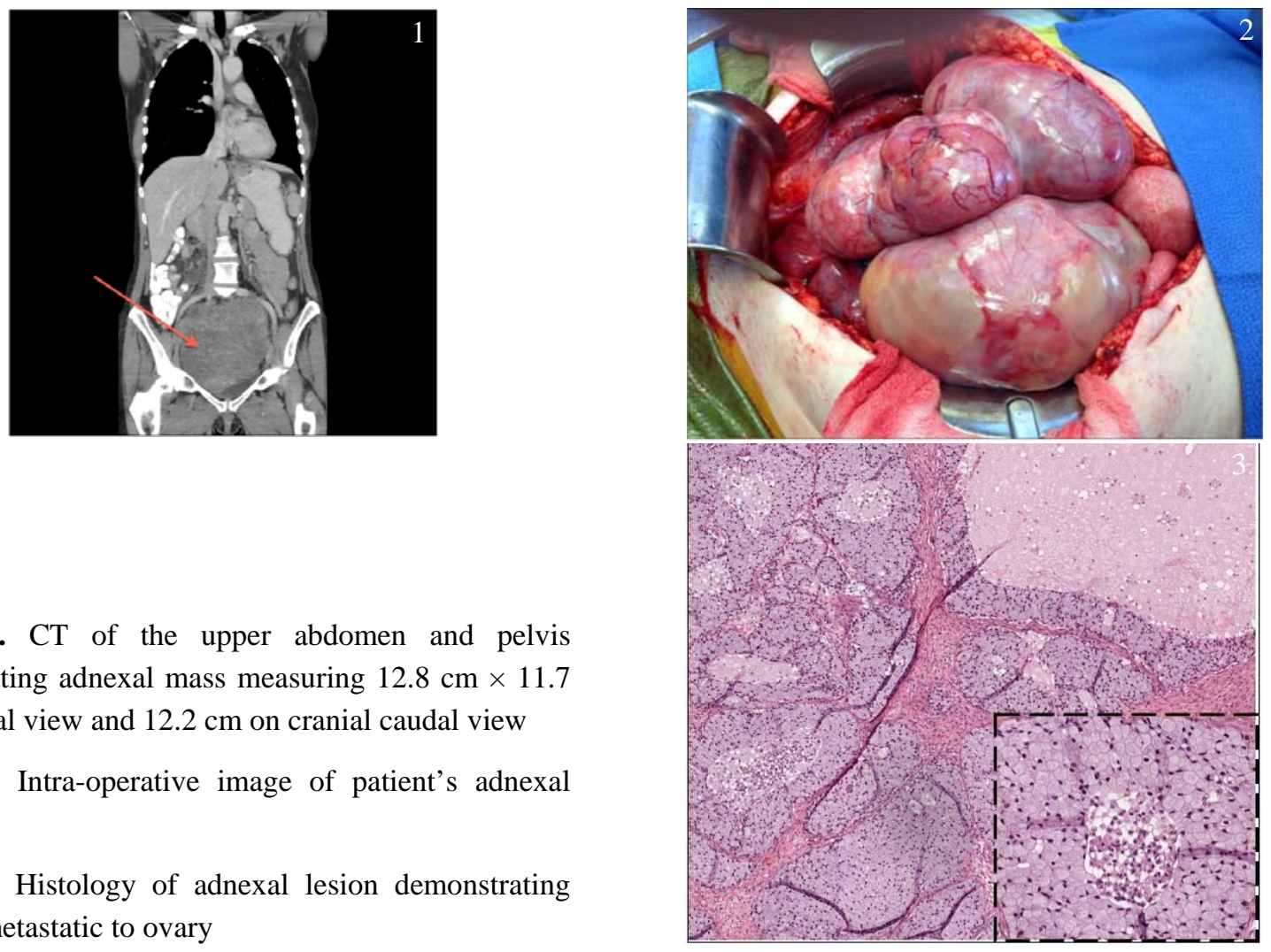

Figure 1. CT of the upper abdomen and pelvis demonstrating adnexal mass measuring $12.8 \mathrm{~cm} \times 11.7$ $\mathrm{cm}$ on axial view and $12.2 \mathrm{~cm}$ on cranial caudal view

Figure 2. Intra-operative image of patient's adnexal mass

Figure 3. Histology of adnexal lesion demonstrating NSCLC metastatic to ovary

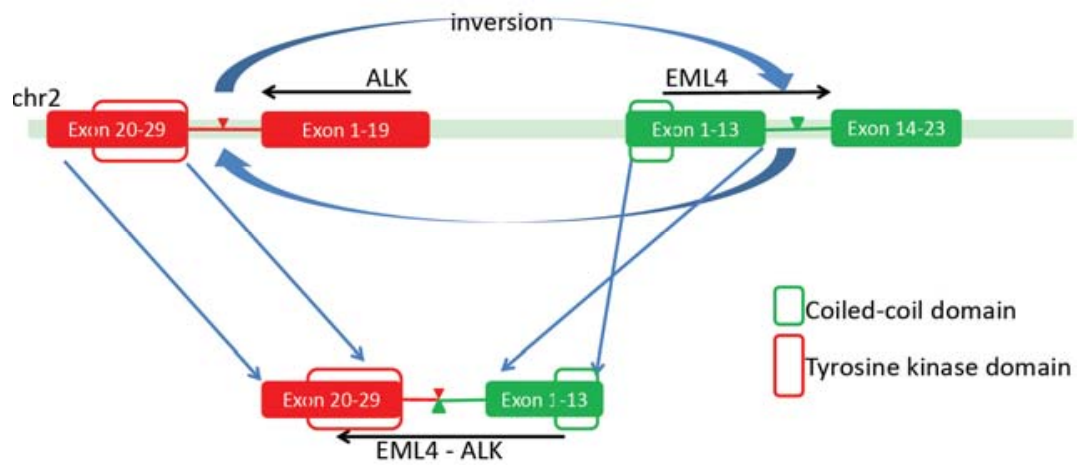

Figure 4. Patient's EML4-ALK translocation. DNA sequencing was performed for 3769 exons of 236 cancer-related genes and for 47 introns of 19 genes that frequently are rearranged in cancer on indexed, adaptor-ligated, hybridizationcaptured libraries using the Illumina HiSeq 2000 (Illumina, Inc., San Diego, Calif). The tumor samples were evaluated for genomic alterations, including base substitutions, short insertions and deletions, amplifications, homozygous deletions, and gene rearrangement ${ }^{[12]}$. 


\section{Discussion}

In this report, we present the case of a 50-year-old female with stage IV EML4-ALK rearrangement positive non-small cell lung cancer (NSCLC) which progresses to adnexal involvement. This serves as another example in the literature of NSCLC metastases to the adnexa in a female with EML4-ALK rearrangement positive disease ${ }^{[1]}$. In NSCLC an estimated $20 \%-50 \%$ of patients will present with metastatic disease ${ }^{[2]}$. Metastatic disease constitutes stage IV NSCLC, however, the scope of illness is broad ranging from diffuse metastatic disease to synchronous solitary metastasis ${ }^{[3,4]}$. The most common sites of NSCLC metastases are the lung, brain, bone, liver, and adrenal glands ${ }^{[4]}$. A wide range of additional locations of NSCLC metastases, albeit much less common, are documented in the literature, including liver, intestine, spleen, adnexa, and pancreas ${ }^{[3]}$. Specifically, the adnexa are a unique location of NSCLC metastasis with limited review in the literature; a malignant ovarian tumor is secondary to metastasis in 5\%-10\% of cases and large case studies looking at site distribution of secondary ovarian neoplasia identified lung cancer as the primary source in $4 \%$ of cases ${ }^{[5]}$. A 2005 review of women at Vancouver General Hospital and Massachusetts General Hospital reported thirty-two cases of lung cancer metastases to the ovary, seven of which were previously reported ${ }^{[5,6]}$. The age of patients spanned from 26-76 with a mean age of $47^{[5]}$. With regards to time course of disease, 53\% had a prior history of lung cancer at time of identification of adnexal metastasis, 31\% demonstrated synchronous lung and ovarian tumors, and 16\% had an identified ovarian tumor up to twenty-six months prior to lung cancer diagnosis ${ }^{[5]}$. The primary lung cancer histology included all four major histologic subtypes: small cell lung cancer $44 \%$, adenocarcinoma $34 \%$, large cell cancer $16 \%$, and 1 squamous cell cancer ${ }^{[5,7]}$. However, until this report, there are no examples of NSCLC metastases to the adnexa in females with ALK fusion positive disease.

This observation prompted a brief chart review of female patients with EML4-ALK positive NSCLC consented for research in our Thoracic Oncology Clinic. Twenty patients met the requirements for chart review: female, NSCLC, EML4-ALK rearrangement positive. Of these twenty charts, sixteen included imaging of the pelvis by computed tomography as part of work-up for systemic disease. One patient had a history of hysterectomy-oophorectomy and was excluded. Therefore fifteen pelvic CT images were considered in our review, five of which were notable for non-specific ovarian enlargement or mass. Further characterization of these findings by ultrasound or MRI was not available to the authors at the time of publication. However, the finding of an ovarian abnormality in 5/15 patients was compelling and as the above case demonstrates, metastases to the adnexa in female patients with ALK rearrangement positive NSCLC should be a consideration during work-up for systemic disease. The theme of unique sites of lung cancer metastases in patients with novel oncogenic mutations such as EML4-ALK rearrangement is also demonstrated in a recent case report of symptomatic gastric metastases in a young female and supported by a recent prospective observational study of 209 persons with stage IV NSCLC evaluated for sites of metastatic disease ${ }^{[8,9]}$. In this study, Doebele and colleagues evaluated the location of metastatic disease in 209 patients with EGFR mutation, ALK rearrangement, KRAS mutation, or triple negative disease and noted a statistically significant relationship between oncogenic profile, location of metastases, and number of metastatic sites. This finding highlights the importance of trends in metastases to help further characterize the behavior of distinct NSCLC oncogene subtypes.

This case also demonstrates that ovarian enlargement or mass are important to follow when evaluating treatment response. The terms sanctuary site and anatomic patterns of failure are used to refer to metastases that do not respond to systemic therapy despite overall reduction in a patient's cancer burden. Most commonly, sanctuary site is implemented in the discussion of brain metastases and failure of systemic treatments due to the blood-brain or blood-tumor barrier ${ }^{[10,11]}$. Explanations for the anatomic pattern of failure observed in this patient include drug penetration and concentration in regions of the body, altered tumor biology of specific tissues, or more traditional mechanisms of acquired resistance such as cancer cell mutations in the kinase domain leading to drug resistance ${ }^{[11]}$. Further exploration into the concept of anatomic failure due to ovarian metastases of ALK fusion positive NSCLC is warranted. 


\section{Conclusion}

The above case reflects metastasis to the adnexa in a female with ALK fusion positive NSCLC. We aim to highlight the relevance of this finding for future understanding of the natural progression of disease in ALK rearranged female patients as well as improved treatment options. Additionally, our observation that one-third of our female clinic patients with ALK rearranged disease have ovarian abnormalities on imaging further supports that metastasis to the ovaries should be a consideration during work-up for systemic disease.

\section{References}

[1] Fujiwara, A., Higashiyama, M., Kanou, T., Tokunaga, T., Okami, J., Kodama, K., et al. Bilateral ovarian metastasis of non-small cell lung cancer with ALK rearrangement. Lung Cancer. 2014; 83(2): 302-304. http://dx.doi.org/10.1016/j.lungcan.2013.11.022

[2] Pfannschmidt, J., Dienemann, H. Surgical treatment of oligometastatic non-small cell lung cancer. Lung Cancer. 2010; 69(3): 251-258. http://dx.doi.org/10.1016/j.lungcan.2010.05.003

[3] Xu, Q., Wang, Y., Liu, H., Meng, S., Zhou, S., Xu, J., et al. Treatment outcome for patients with primary NSCLC and synchronous solitary metastasis. Clin Transl Oncol. 2013; 15(10): 802-809. http://dx.doi.org/10.1007/s12094-013-1008-2

[4] Schuchert, M. J., Luketich, J. D. Solitary sites of metastatic disease in non-small cell lung cancer. Curr Treat Options Oncol. 2003; 4(1): 65-79. PMid: 12525281. http://dx.doi.org/10.1007/s11864-003-0033-8

[5] Irving, J. A., Young, R. H. Lung carcinoma metastatic to the ovary: a clinicopathologic study of 32 cases emphasizing their morphologic spectrum and problems in differential diagnosis. Am J Surg Pathol. 2005; 29(8): 997-1006. PMid: 16006793.

[6] Young, R. H., Scully, R. E. Ovarian metastases from cancer of the lung: problems in interpretation--a report of seven cases. Gynecol Oncol. 1985; 21(3): 337-350. http://dx.doi.org/10.1016/0090-8258(85)90272-0

[7] Young, R. H.. From Krukenberg to today: the ever present problems posed by metastatic tumors in the ovary. Part II. Adv Anat Pathol. 2007; 14(3): 149-177. http://dx.doi.org/10.1097/PAP.0b013e3180504abf

[8] Diem, S., Fruh, M., Rodriguez, R., Liechti, P., Rothermundt, C. EML4-ALK-Positive Pulmonary Adenocarcinoma with an Unusual Metastatic Pattern: A Case Report. Case Rep Oncol. 2013; 6(2): 316-319. http://dx.doi.org/10.1159/000352086

[9] Doebele, R. C., Lu, X., Sumey, C., Maxson, D. A., Weickhardt, A. J., Oton, A. B., Camidge, D. R. Oncogene status predicts patterns of metastatic spread in treatment-naive nonsmall cell lung cancer. Cancer. 2012; 118(18): 4502-4511. http://dx.doi.org/10.1002/cncr.27409

[10] Palmieri, D., Chambers, A. F., Felding-Habermann, B., Huang, S., Steeg, P. S. The biology of metastasis to a sanctuary site. Clin Cancer Res. 2007; 13(6): 1656-1662. http://dx.doi.org/10.1158/1078-0432.CCR-06-2659

[11] Chun, S. G., Choe, K. S., Iyengar, P., Yordy, J. S., Timmerman, R. D. Isolated central nervous system progression on Crizotinib: an Achilles heel of non-small cell lung cancer with EML4-ALK translocation? Cancer Biol Ther. 2012; 13(14): 1376-1383. http://dx.doi.org/10.4161/cbt.22255

[12] Frampton, G. M., Fichtenholtz, A., Otto, G. A., Wang, K., Downing, S. R., He, J., et al. Development and validation of a clinical cancer genomic profiling test based on massively parallel DNA sequencing. Nat Biotechnol. 2013; 31(11): $1023-1031$. http://dx.doi.org/10.1038/nbt.2696 